\title{
Molecular subtype-specific efficacy of anti-EGFR therapy in colorectal cancer is dependent on the chemotherapy backbone
}

\author{
Sanne ten Hoorn (D) $^{1,2}$, Dirkje W. Sommeijer ${ }^{1,3,4}$, Faye Elliott ${ }^{5}$, David Fisher ${ }^{6}$, Tim R. de Back ${ }^{1,2}$, Anne Trinh ${ }^{7,8}$, Lianne Koens ${ }^{9}$, \\ Tim Maughan ${ }^{10}$, Jenny Seligmann $D^{5}$, Matthew T. Seymour ${ }^{5}$, Phil Quirke ${ }^{5}$, Richard Adams ${ }^{11}$, Susan D. Richman ${ }^{5}$, \\ Cornelis J. A. Punt ${ }^{3,12}$ and Louis Vermeulen (iD ${ }^{1,2,3}$ 凶凶
}

(c) The Author(s) 2021

\begin{abstract}
BACKGROUND: Patient selection for addition of anti-EGFR therapy to chemotherapy for patients with RAS and BRAF wildtype metastatic colorectal cancer can still be optimised. Here we investigate the effect of anti-EGFR therapy on survival in different consensus molecular subtypes (CMSs) and stratified by primary tumour location.

METHODS: Retrospective analyses, using the immunohistochemistry-based CMS classifier, were performed in the COIN (first-line oxaliplatin backbone with or without cetuximab) and PICCOLO trial (second-line irinotecan with or without panitumumab). Tumour tissue was available for 323 patients (20\%) and 349 (41\%), respectively.

RESULTS: When using an irinotecan backbone, anti-EGFR therapy is effective in both CMS2/3 and CMS4 in left-sided primary tumours (progression-free survival (PFS): HR $0.44,95 \% \mathrm{Cl} 0.26-0.75, P=0.003$ and HR $0.12,95 \% \mathrm{Cl} 0.04-0.36, P<0.001$, respectively) and in CMS4 right-sided tumours (PFS HR $0.17,95 \% \mathrm{Cl} 0.04-0.71, P=0.02$ ). Efficacy using an oxaliplatin backbone was restricted to left-sided CMS2/3 tumours (HR 0.57, 95\% Cl 0.36-0.96, $P=0.034$ ).

CONCLUSIONS: The subtype-specific efficacy of anti-EGFR therapy is dependent on the chemotherapy backbone. This may provide the possibility of subtype-specific treatment strategies for a more optimal use of anti-EGFR therapy.
\end{abstract}

British Journal of Cancer (2021) 125:1080-1088; https://doi.org/10.1038/s41416-021-01477-9

\section{INTRODUCTION}

Since the introduction of targeted agents, anti-VEGF and antiEGFR therapy have become part of the standard treatment arsenal for patients with metastatic colorectal cancer (mCRC). Anti-EGFR agents (cetuximab, panitumumab) may be given as monotherapy in chemorefractory patients, but are usually combined in earlier lines (first or second line) with chemotherapy, with either an oxaliplatin or irinotecan backbone, which are both considered effective and safe regimens $[1,2]$.

The efficacy of anti-EGFR therapy was shown to be restricted to the subgroup of patients with RAS or BRAFV600E wildtype tumours, as no benefit was observed in patients with tumours that harbour these mutations [3, 4]. Anti-EGFR therapy was even associated with a detrimental effect in patients with KRAS mutant tumours [5-7]. Patient selection for anti-EGFR therapy was further improved by taking into account the sidedness of the primary tumour, since patients with right-sided primary tumours do not benefit from the addition of anti-EGFR to chemotherapy [8].
However, more recent data caution against the absolute use of this criterion $[9,10]$. This stresses the need for further stratification and patient selection beyond RAS/BRAF mutations and tumour sidedness.

Recent work on molecular subtyping has demonstrated its predictive value for anti-EGFR therapies, and thus diagnostic utility in optimising selection criteria. The consensus molecular subtypes (CMSs) capture the biological heterogeneity in colorectal cancer by recognising four distinct subtypes [11]: with CMS1 characterised by microsatellite instability, strong immune activation and BRAF-mutations; CMS2 is an epithelial subtype with high chromosomal instability and prominent WNT and MYC signalling activation; CMS3 is also an epithelial subtype with metabolic dysregulation and is enriched for KRAS-mutations; and CMS4 represents a mesenchymal subtype with marked TGF- $\beta$ activation and abundant stromal content. Whilst patients with epithelial CMS2/3 tumours benefit from anti-EGFR agents given as monotherapy or combined with an oxaliplatin-based chemotherapy

\footnotetext{
${ }^{1}$ Amsterdam UMC, University of Amsterdam, LEXOR, Center for Experimental and Molecular Medicine, Cancer Center Amsterdam, Amsterdam, The Netherlands. ${ }^{2}$ Oncode

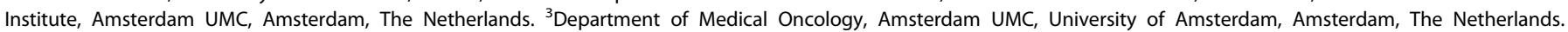

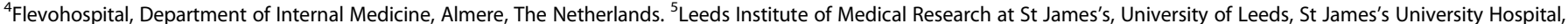

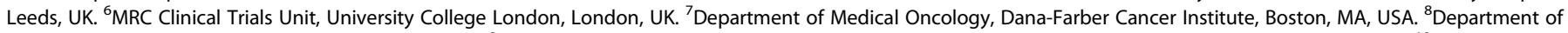

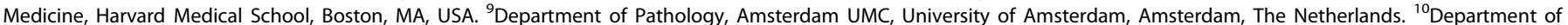

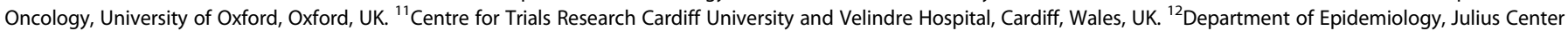

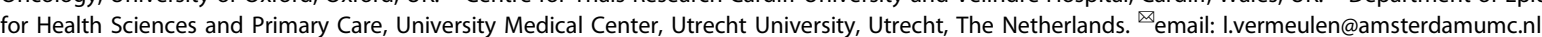

Received: 30 March 2021 Revised: 8 June 2021 Accepted: 30 June 2021

Published online: 12 July 2021 
a

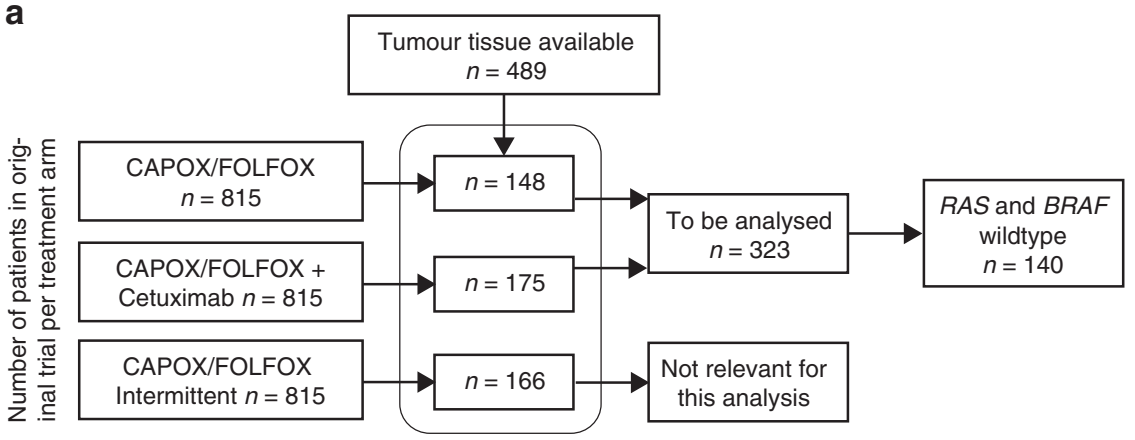

b

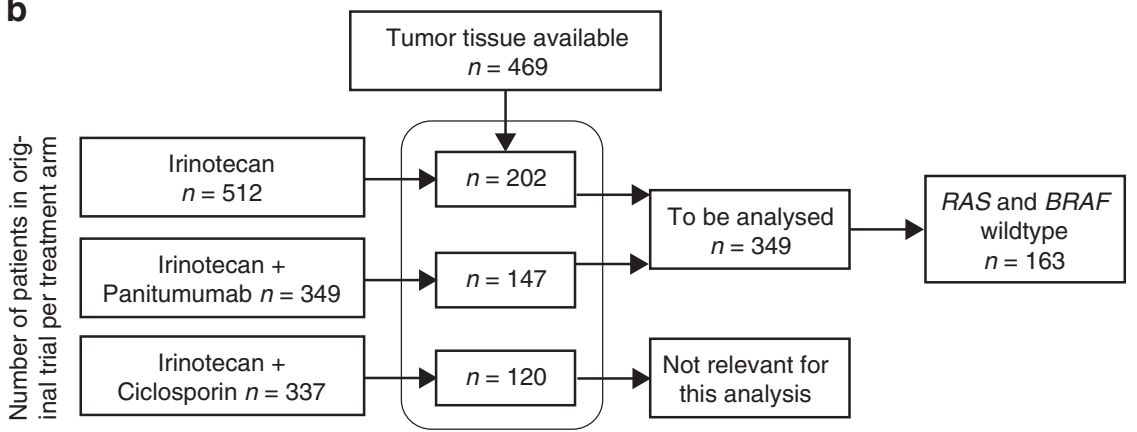

Fig. 1 Study flow diagram. Overview of included samples for the COIN trial (a) and PICCOLO trial (b). CAPOX, capecitabine and oxaliplatin; FOLFOX, 5-fluorouracil and oxaliplatin.

regimen [12-14], patients with CMS4 tumours appear to have no or even a detrimental effect when anti-EGFR is added to an oxaliplatin-based regimen [13, 14]. In contrast, patients with mesenchymal CMS4 mCRC have a significant survival benefit when anti-EGFR is added to FOLFIRI, whereas for patients with CMS2 tumours no such benefit was seen [15]. An analysis on the differences between the CALB/SWOG 80405 and FIRE-3 studies also suggested that cetuximab activity is synergistic with irinotecan in all CMS subgroups, and with oxaliplatin only in CMS2 and CMS3 [16]. Based on these studies we hypothesised that anti-EGFR therapy has a subtype specific effect, which is dependent on the chemotherapy backbone used.

To further investigate the possible differential effect of backbone regimens in combination with anti-EGFR therapy for the different molecular subtypes we stratified patients enrolled in two prospective clinical trials, the COIN and the PICCOLO trials $[17,18]$. The treatment effects of either a first-line oxaliplatinbased (COIN trial) or a second-line irinotecan backbone (PICCOLO trial) on anti-EGFR activity stratified by CMSs were studied. We hypothesised that anti-EGFR treatment is most effective when added to an oxaliplatin backbone in patients with CMS2/3 tumours in the COIN trial, while patients with mesenchymal CMS4 benefit most from anti-EGFR added to an irinotecan backbone as given in the PICCOLO trial. Furthermore, we performed exploratory analyses into differing effects according to tumour sidedness and CMSs to further define anti-EGFR therapy efficacy.

\section{METHODS}

\section{Patients}

Details on both trial protocols have been previously reported $[17,18]$. In short, the randomised controlled, multicenter, COIN trial was conducted between 2005 and 2008. Patients without previous chemotherapy for $\mathrm{mCRC}$ were randomly assigned to oxaliplatin and fluoropyrimidine chemotherapy (CAPOX (capecitabine and oxaliplatin) or FOLFOX (5-fluorouracil and oxaliplatin)), the same combination plus cetuximab, or intermittent chemotherapy. No selection based on mutation status was done.

The PICCOLO trial was a multicenter, randomised controlled trial in the second-line treatment of mCRC. Patients were included between 2006 and 2010 to a three-arm design of second-line therapy of irinotecan, irinotecan plus ciclosporin and irinotecan plus panitumumab. From June 10, 2008 panitumumab randomisation was restricted to patients with KRASC.12,13,61 wildtype tumours.

Primary endpoint of both studies was overall survival (OS), with secondary endpoints of progression-free survival (PFS), tumour response (RECIST) and toxicity. For current analyses, only RAS (for both trials: KRASc.12,13,61 and NRASC.12,61, additional for the PICCOLO trial: KRASC.146 and NRASc.13) and BRAFc.600 wildtype patients of the CAPOX/FOLFOX and CAPOX/FOLFOX with cetuximab treatment arms from the COIN trial and irinotecan and irinotecan with panitumumab arm from the PICCOLO trial were included.

Right-sided primary tumours were defined as tumours located proximal from the splenic flexure, left-sided tumours as tumours arising in or distal from the splenic flexure.

\section{CMS Classification}

Tumour tissue from the primary tumour was collected for both trials from all available patients. For each primary tumour three or four cores were available on a tissue microarray (TMA) on a 4- $\mu$ m-thick section slide. Tumours were stratified into the different consensus molecular subtypes using the previously developed immunohistochemistry (IHC)-based classifier [14, 19]. CMS1 patients were first classified using mismatch repair (MMR) protein expression status, identified by IHC of four markers (MLH1, MSH2, MSH6 and PMS2). Tumours with loss of expression of one of these markers were considered MMR deficient. Next, TMA slides were stained for five markers (CDX2, FRMD6, HTR2B, ZEB1 and KER) and classified into epithelial (CMS2/3) or mesenchymal subtype (CMS4) using the published image analysis pipeline and CMS-IHC classifier [14]. A probability of $>60 \%$ was used for a core to be classified as mesenchymal, and a tumour was classified as CMS4 if at least one core was identified as mesenchymal.

\section{Statistical analysis}

Stata version 15 was used for statistical analyses. Baseline patient characteristics were compared between the different subtypes using 
Pearson Chi-squared tests for categorical variables where the count was $>5$ in a cell and Fishers exact tests otherwise. Kruskal-Wallis tests were used for continuous variables. For calculation of $P$-values, unknowns were excluded.

Time-to-event curves for PFS and OS were calculated using the Kaplan-Meier method. Hazard ratios (HRs), 95\% confidence intervals (Cls) and $P$-values were estimated using cox proportional Hazards models. Response data were compared between treatment groups using logistic regression and estimating odds ratios (ORs). All presented HRs and ORs for the main and sensitivity analysis are adjusted for age, sex and WHO performance status. $P$-values were two-sided and an arbitrary $5 \%$ cut-off was used for statistical significance. $R$ software version 4.0 .5 was used for $P$ value multiple testing correction using the Benjamini-Hochberg procedure, with an alpha of 0.05 [20]. We corrected $P$-values per independent analysis, i.e., for the total cohorts, left-sided and right-sided tumours. Adjusted $P$ values are indicated in the figure legends.

\section{RESULTS \\ Patient characteristics}

Of 1630 COIN trial patients enrolled in the treatment arms of interest, tumour tissue for CMS classification was available for 323 patients (19.8\%), of which $140(43.3 \%)$ were RAS and BRAF wildtype. For the PICCOLO trial, of 861 patients enrolled in the treatment arms of interest for $349(40.5 \%)$ tumour tissue was available for classification with 163 (46.7\%) being RAS and BRAF wildtype (Fig. 1). All patients with tumour tissue available were classified into either CMS2/3 or CMS4 using a previously developed and validated immunohistochemical assay [14, 19]. For both cohorts the classified samples were representative for the total study population, but had improved PFS and OS (nonsignificant) with a higher proportion of resected primary tumours (Supplementary Table 1). This is inherently linked to the method of CMS classification used, as this requires sufficient tumour tissue for staining. In the RAS and BRAF wildtype cohort, the treatment arms were well balanced, apart from the primary tumour location in the PICCOLO trial, in which case the proportion of right-sided tumours was higher in the control (irinotecan) arm (Supplementary Table 2).

\section{CMS classification}

In the classified wildtype cohort, 37\% (52/140 COIN) and 31\% (51/ 163 PICCOLO) of patients were classified as CMS4 (Table 1). CMS1 was excluded in the analysis due to low numbers in both cohorts $(n=4$ and $n=2)$, in line with the notion that MMR deficient cancers are rare in $\mathrm{mCRC}$ [21]. Patients with CMS4 cancers were younger compared to CMS2/3 in the COIN trial (mean age 60.8 $(\mathrm{SD}=10.2)$ versus $64.9(\mathrm{SD}=8.4), P=0.037)$, and $\mathrm{CMS} 2 / 3$ cancers were more frequently left-sided in the PICCOLO trial $(45 \%$ versus $27 \%, P=0.04$ ) (Table 1 ). In the COIN trial CMS2/3 patients have a (non-significant) longer overall survival (22.3 versus 15.7 months, $P=0.07$ ) compared to CMS4, for the PICCOLO trial on second-line anti-EGFR therapy there was no difference in survival between the epithelial and mesenchymal subtypes.

\section{Efficacy of anti-EGFR in CMS subtypes}

When assessing the treatment response in the RAS and $B R A F$ wildtype cohort there was a clear difference in response to anti-EGFR for the CMS4 tumours between both trials. When added to irinotecan there was a significantly longer PFS in CMS4 (HR 0.26, 95\% Cl 0.13-0.52, $P<0.001$ ) (Fig. 2a), but when added to CAPOX/FOLFOX there was no beneficial effect of the addition of cetuximab (HR 1.68, 95\% Cl 0.91-3.10, $P=0.10$ ) (Fig. 2b). For CMS2/3 patients there was also a (non-significant) benefit for anti-EGFR addition to irinotecan in the PICCOLO trial (HR 0.67, $95 \% \mathrm{Cl}$ 0.44-1.01, $P=0.06$ ) (Fig. 2c), and a (non-significant) PFS benefit when added to CAPOX/FOLFOX in the COIN trial (HR $0.69,95 \% \mathrm{Cl} 0.43-1.11, P=0.12$ ) (Fig. 2d). No difference in OS was observed.
As the combination of capecitabine-based chemotherapy and cetuximab was shown to be more toxic and, therefore, less effective compared to 5-fluorouracil-based chemotherapy and cetuximab $[17,22,23]$, a sensitivity analysis for the COIN trial with patients receiving either CAPOX or FOLFOX was performed. Among wildtype patients, $63 \%(n=86)$ was treated with either CAPOX or the combination of CAPOX with cetuximab. For CMS2/3 patients there was indeed a better outcome when cetuximab was added to FOLFOX, though this was not significant (PFS HR 0.45, $95 \% \mathrm{Cl} 0.17-1.20, P=0.11$; OS HR $0.48,95 \% \mathrm{Cl} 0.17-1.31, P=0.15)$ (Supplementary Fig. 1A).

Analysis of the response data convey comparable results, with response only observed in CMS4 when panitumumab was added to irinotecan (OR 8.52, 95\% Cl 1.69-43.05, $P=0.01$ ) (Table 2). In CMS2/3 there was also a significantly better response when panitumumab was added to irinotecan (OR 4.27, $95 \% \mathrm{Cl} 1.66-11.00, P=0.003)$, no difference was observed to the addition of cetuximab to CAPOX/FOLFOX (OR $1.72,95 \% \mathrm{Cl}$ $0.60-4.95, P=0.31)$.

\section{Effect of primary tumour location}

In the combined RAS and BRAF wildtype cohort the majority of the tumours were left-sided (75\%; COIN: $81 \%$ and PICCOLO: 69\%). The distribution of the CMSs was similar in both trials and there was no difference between left- and right-sided tumours ((Left: CMS2/3 65\%, CMS4 35\%; Right: CMS2/3: 64\%, CMS4: 36\%; $X^{2}=0.077, P=$ 0.78) (Supplementary Table 3).

In left-sided tumours, a clear difference between the subtypes in efficacy of anti-EGFR with the different backbones was observed, with CMS2/3 having a significant PFS benefit from the addition of anti-EGFR with both chemotherapy backbones (CAPOX/FOLFOX HR 0.57, 95\% Cl 0.36-0.96, $P=0.034$; Irinotecan HR $0.44,95 \% \mathrm{Cl} 0.26-0.75, P=0.003)$. In CMS4 there was only a significant PFS benefit for anti-EGFR when added to irinotecan (HR $0.12,95 \% \mathrm{Cl} 0.04-0.36, P<0.001$ ). When anti-EGFR was added to CAPOX/FOLFOX in CMS4 tumours even a detrimental effect was seen (HR 2.76, 95\% Cl 1.27-6.01, $P=0.006$ ) (Fig. 3 and Supplementary Fig. 1B).

For right-sided tumours a significant PFS and OS benefit was observed for CMS4 tumours when anti-EGFR was added to irinotecan as second line chemotherapy for both PFS (HR 0.17, $95 \% \mathrm{Cl} 0.04-0.71, P=0.02$ ) and OS (HR $0.20,95 \% \mathrm{Cl} 0.05-0.83$, $P=0.03$ ) (Fig. 4 and Supplementary Fig. 1C). No benefit of antiEGFR was observed in right-sided CMS2/3 tumours. A summary of our results with possible implications for treatment recommendations based on the CMSs and tumour sidedness is shown in Supplementary Fig. 2.

\section{DISCUSSION}

In this retrospective study of two trial cohorts, we show a differential effect of anti-EGFR therapy in the main CMS subtypes when combined with different chemotherapy backbones. The largest benefit of anti-EGFR was observed in patients with mesenchymal CMS4 tumours when combined with an irinotecan backbone. This effect was seen in both left and right-sided primary tumours. For epithelial CMS2/3 tumours there was a benefit from the addition of anti-EGFR when added to both oxaliplatin and irinotecan-based chemotherapy, which was only observed in leftsided primary tumours.

Our results are in line with others, who showed a PFS and OS survival benefit effect from anti-EGFR for CMS4 when added to an irinotecan-based first-line treatment regimen $[15,16]$. The high disease control rate in CMS4 but also in CMS2 when antiEGFR is combined with irinotecan was also shown in a recent publication, $93.3 \%$ and $76.9 \%$ for CMS2 and CMS4, respectively [24]. The CALGB/SWOG 80405 study did not show a survival difference for the addition of either bevacizumab or cetuximab 
Table 1. Baseline and survival characteristics for the molecular subtypes in RAS and BRAF wildtype cohort.

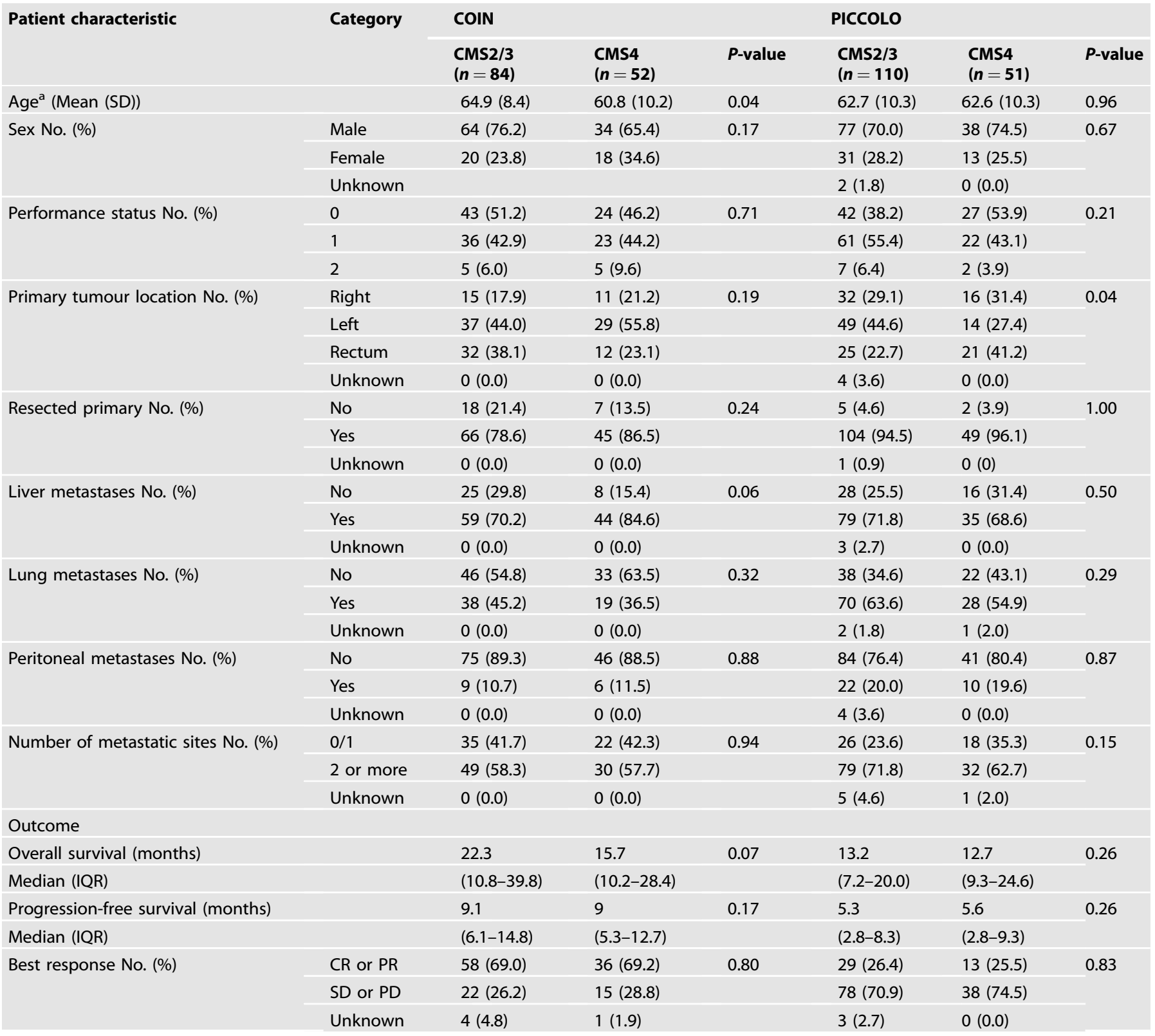

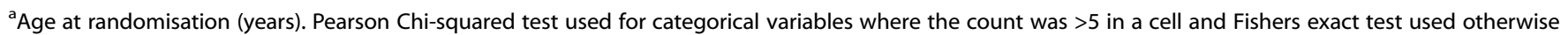
Kruskal-Wallis test used for continuous variables. Unknowns were excluded for testing variables. Log-rank test was used for survival outcomes. CR or PR, complete or partial response; N, number of SD or PD, patients; SD, standard deviation; stable disease or progressive disease.

to chemotherapy in CMS4 tumours [13]. Unfortunately, numbers to perform separate analyses on patients receiving either a FOLFOX or FOLFIRI chemotherapy backbone are relatively low.

Various clinical studies support the notion that the efficacy of anti-EGFR antibodies is restricted to left-sided tumours [8, 25, 26]. However, a recently updated meta-analysis indicated that antiEGFR therapies could remain an option for patients with RAS wildtype right-sided tumours, as this significantly improved PFS and therapy response in both left- and right-sided tumours [9]. This finding is in line with the here reported results, which show that patients with both left- and right-sided CMS4 tumours benefit from the combination of irinotecan and panitumumab (Figs. 3 and 4). Therefore, anti-EGFR may potentially be considered in patients with right-sided CMS4 tumours.
Several preclinical studies have shown a synergistic effect between anti-EGFR therapy and irinotecan [27-29]. Tumour cell exposure to irinotecan leads to resistance through upregulation of EGFR signalling. Anti-EGFR overcomes this resistance through downregulation of the EGFR pathway which is upregulated by irinotecan $[28,30]$, explaining the benefit observed in irinotecanrefractory $\mathrm{mCRC}$ [31]. Another proposed mechanism underlying the enhanced tumour response of the combination of these two agents is a cetuximab induced suppression of mammalian heat shock protein 27 (HSP27), which is reported to be involved in irinotecan resistance, through blocking the JAK/STAT signalling pathway in RAS wildtype CRC cells [27].

There is some evidence that irinotecan-based regimens have a higher overall response rate (ORR) and improved PFS when 


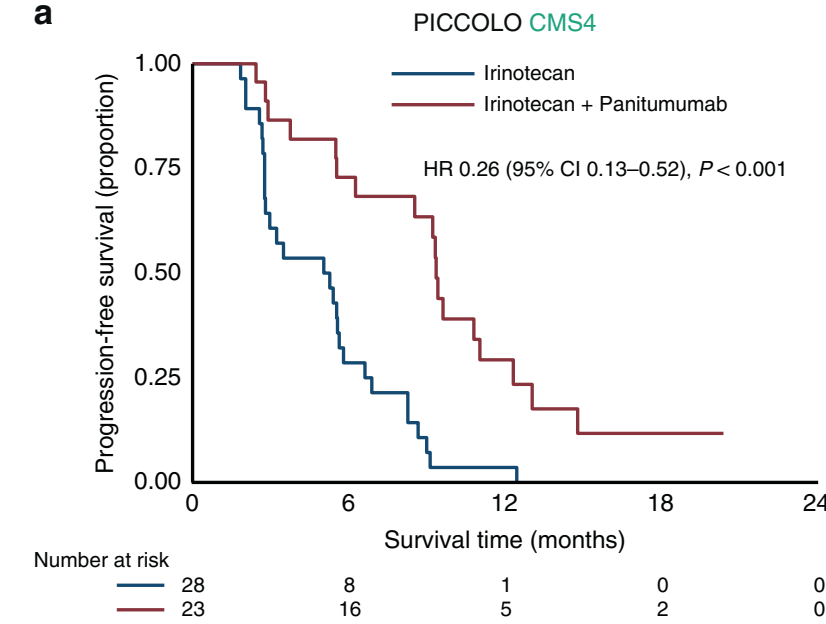

C

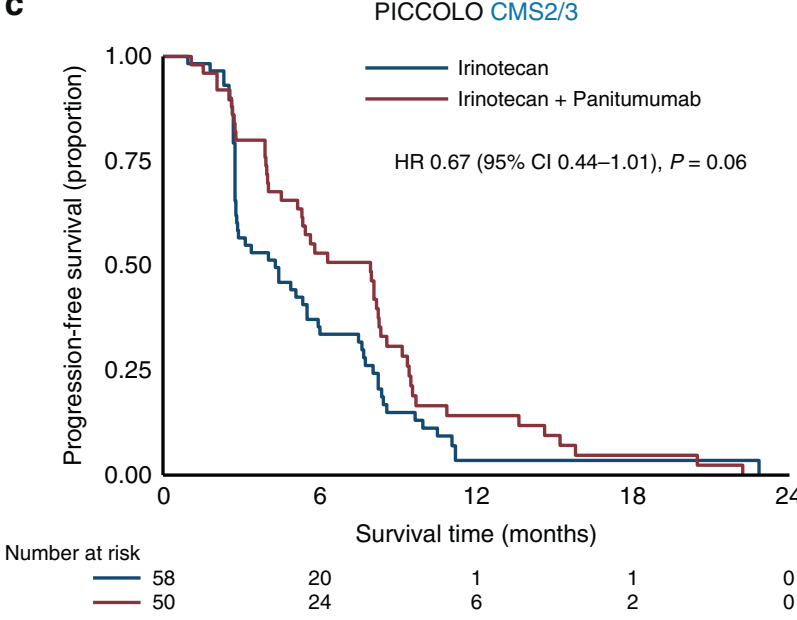

b

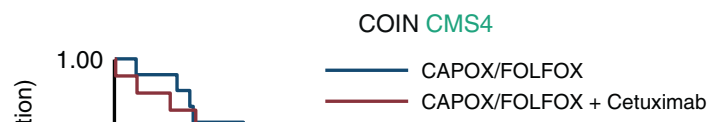

\section{4}

Number at risk

Number at risk 27

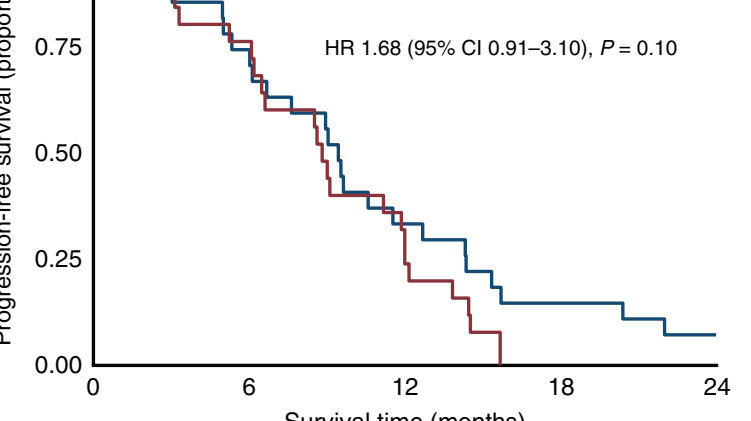

d
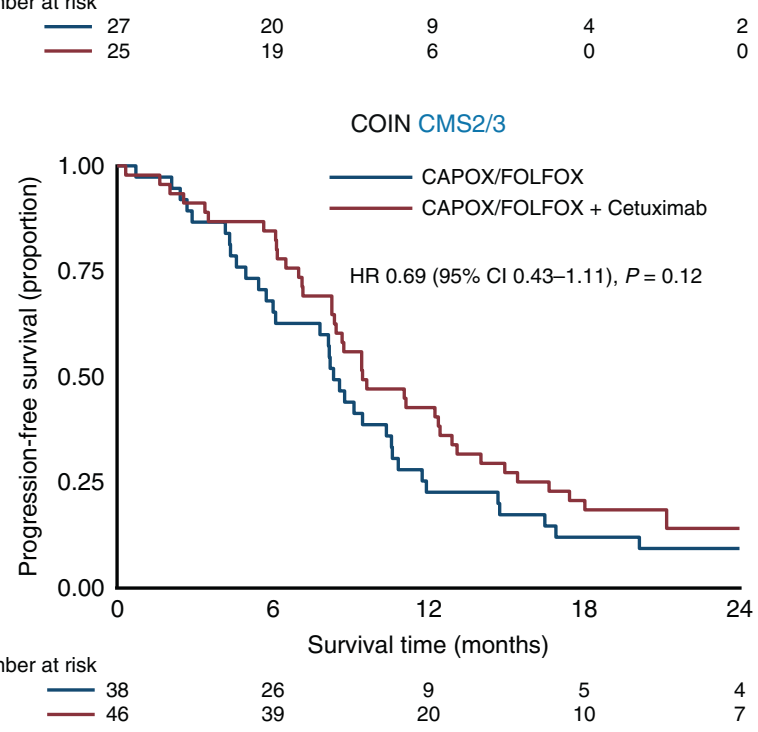

Fig. 2 Molecular subtype-specific efficacy of anti-EGFR. Progression-free survival for CMS4 in the PICCOLO trial (a) and the COIN trial (b) and for CMS2/3 in the PICCOLO trial (c) and the COIN trial (d). HRs are adjusted for age, sex and WHO performance status. CAPOX capecitabine and oxaliplatin, FOLFOX 5-fluorouracil and oxaliplatin, HR hazard ratio. P-values adjusted for multiple testing: 0.12 (a), 0.10 (b), 0.12 (c), 0.004 (d).

Table 2. Best response ${ }^{a}$ for subtype-specific anti-EGFR efficacy.

\begin{tabular}{|c|c|c|c|c|c|c|c|c|c|}
\hline \multirow[t]{3}{*}{ Trial } & \multirow[t]{3}{*}{ Treatment } & \multicolumn{4}{|l|}{ CMS2/3 } & \multicolumn{4}{|l|}{ CMS4 } \\
\hline & & \multicolumn{2}{|c|}{ CR or PR No. (\%) } & \multirow[t]{2}{*}{ OR $(95 \% \mathrm{Cl})$} & \multirow[t]{2}{*}{$P$-value } & \multicolumn{2}{|c|}{ CR or PR No. (\%) } & \multirow[t]{2}{*}{ OR (95\% Cl) } & \multirow[t]{2}{*}{$P$-value } \\
\hline & & Yes & No & & & Yes & No & & \\
\hline \multirow{2}{*}{ COIN } & CAPOX/FOLFOX & $35(77.8)$ & $10(22.2)$ & 1.72 & \multirow{2}{*}{0.31} & $17(70.8)$ & $7(29.2)$ & 1.05 & \multirow{2}{*}{0.95} \\
\hline & + Cetuximab & & & $(0.60-4.95)$ & & & & $(0.28-3.96)$ & \\
\hline PICCOLO & + Panitumumab & & & $(1.66-11.00)$ & 0.003 & & & $(1.69-43.05)$ & 0.01 \\
\hline
\end{tabular}

${ }^{a}$ Best response: $C R / P R$ versus SD/PD/Death. Only deaths which occurred within 12 weeks were included. ORs are adjusted for age, sex and WHO performance status. CAPOX, capecitabine and oxaliplatin (CAPOX/FOLFOX); CR, complete response; FOLFOX, 5-fluorouracil and oxaliplatin; OR, odds ratio; PR, partial response; SD, stable disease; PD, progressive disease.

combined with anti-EGFR in comparison to the combination with an oxaliplatin-based regimen [32,33]. However, this may also be explained by the negative interaction between capecitabine in CAPOX regimens and anti-EGFR, as there is no difference in effectiveness when comparing the addition of anti-EGFR to either FOLFOX or FOLFIRI $[22,23]$.
Irinotecan-based treatment regimens, in general, appear to be more effective in CMS4 tumours when compared to oxaliplatinbased therapies [24, 34-36]. Moreover, preclinical studies in CMS4 cell lines and stem-like subtype tumours in patients, which are related to CMS4, show a marked response to topoisomerase inhibitors [37, 38]. However, the reason why mesenchymal 
COIN CMS2/3

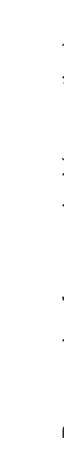

b

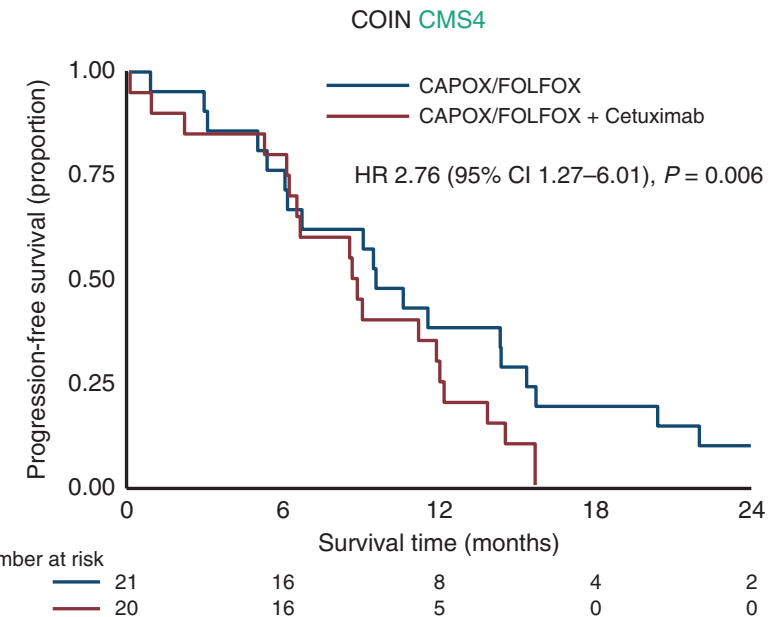

d

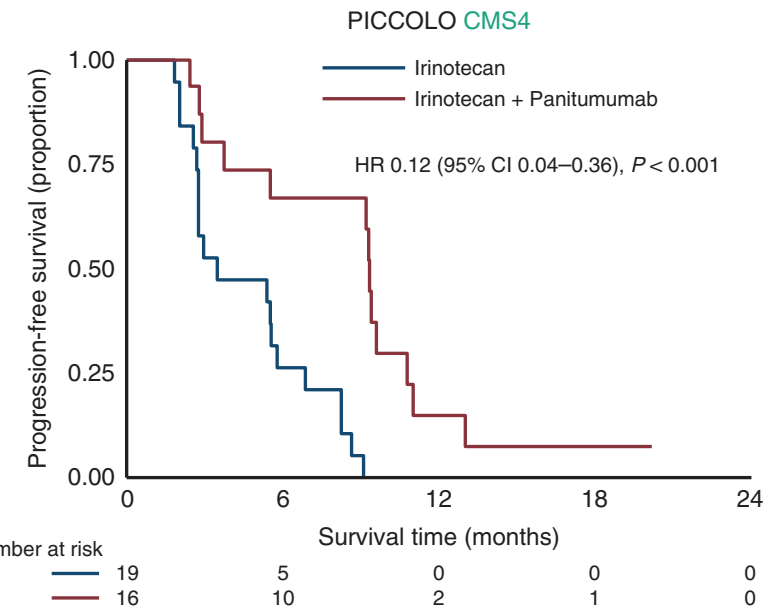

Fig. 3 Molecular subtype-specific efficacy of anti-EGFR in left-sided tumours. Progression-free survival in the COIN trial for CMS2/3 (a) and CMS4 (b) and the PICCOLO trial for CMS2/3 (c) and CMS4 (d). HRs are adjusted for age, sex and WHO performance status. CAPOX capecitabine and oxaliplatin, FOLFOX 5-fluorouracil and oxaliplatin, HR hazard ratio. $P$-values adjusted for multiple testing: 0.034 (a), 0.008 (b), 0.006 (c), 0.004 (d).

tumours seem to be more susceptible to an irinotecan-based chemotherapy regimen is not yet understood. A possible mechanism of the antagonistic action of oxaliplatin in combination with anti-EGFR could be the fibroblast rich tumour microenvironment of CMS4 cancers, that increases the effects of oxaliplatin induced cytokines, which might subsequently antagonise the antitumor effects of cetuximab and the cetuximaboxaliplatin synergy [16].

In the current study we show that patients with CMS2/3 tumours may benefit from anti-EGFR therapy combined with either an irinotecan or oxaliplatin backbone. The effects seen in the COIN trial were however small, which is likely explained by the fact that the majority of patients in this study were treated with CAPOX and cetuximab, notably this regimen is currently not recommended for its increased toxicity and lack of efficacy $[17,22,23]$. A sensitivity analysis showed a much better response when cetuximab was added to FOLFOX as compared to CAPOX (Supplementary Fig. 1A).

In both the COIN and PICCOLO trials a benefit of the addition of anti-EGFR was seen only for PFS, but not for OS, with an exception for the right-sided CMS4 tumours which benefited from the addition of anti-EGFR to irinotecan in both PFS and OS. The PFS is a direct measure of the treatment effect of the study, while $O S$ is the result of the cumulative survival after all the lines of treatment that the patients underwent. Lack of overall survival benefit in the COIN trial was attributed by the authors to the combination of including patients with more advanced CRC and the more toxic effects of the combination of CAPOX and cetuximab [17]. For the FOLFOX treated patients we have indeed shown a trend towards OS benefit when anti-EGFR is added (Supplementary Fig. 1A). For the PICCOLO trial also only a PFS benefit was detected for the wildtype cohort. An inferior survival after relapse for the panitumumab treated patients was observed, especially for the population with a mutation (either KRAS, BRAF, NRAS or PIK3CA), possibly due to accelerated tumour growth caused by the anti-EGFR therapy [18]. In this study, however, we did see an OS benefit for the addition of anti-EGFR to panitumumab in right-sided CMS4 patients (Supplementary Fig. 1C).

We detected a trend towards OS benefit in CMS2/3 in the COIN trial, and no difference in survival between CMS2/3 and CMS4 in the PICCOLO trial. When looking at the total population, CMS4 confers the worst prognosis, because often these tumours present at a higher stage [21]. In $\mathrm{mCRC}$ the difference in survival between CMS2 and CMS4 is less pronounced, and worse survival is described for CMS1 [11]. Although numbers of CMS1 were low in this subpopulation, we did see indeed the lowest survival time for CMS1 in both trials (data not shown). 
a

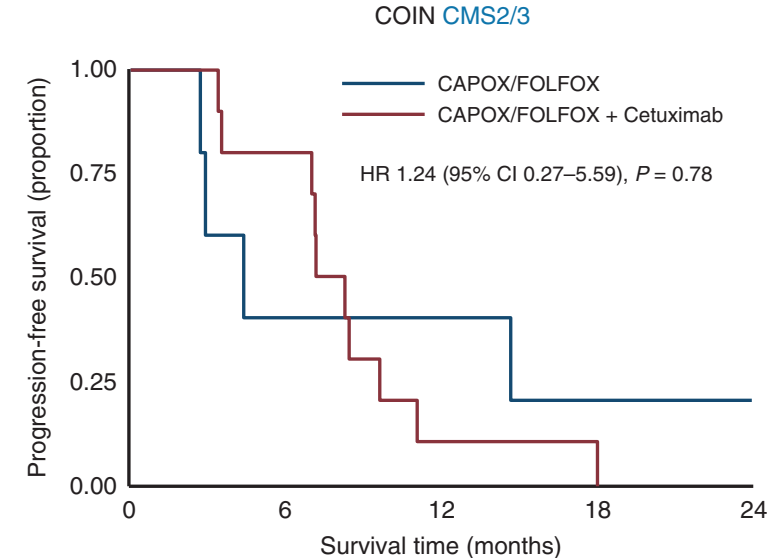

b

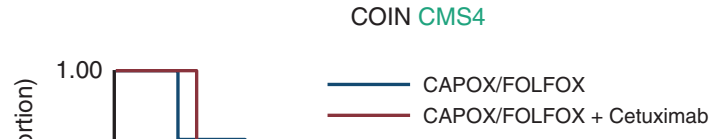

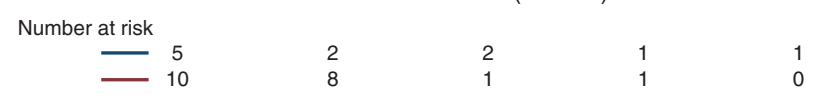

c

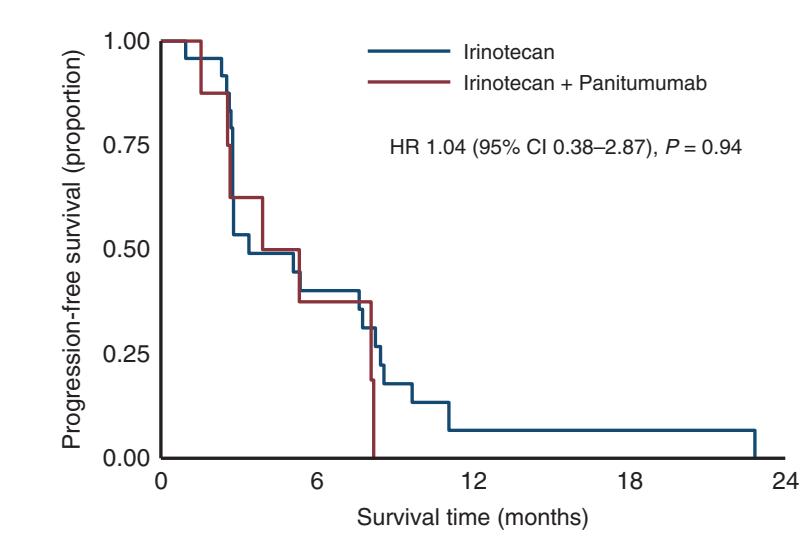

PICCOLO CMS2/3

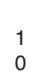

Number at risk

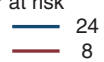

9
2

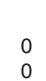

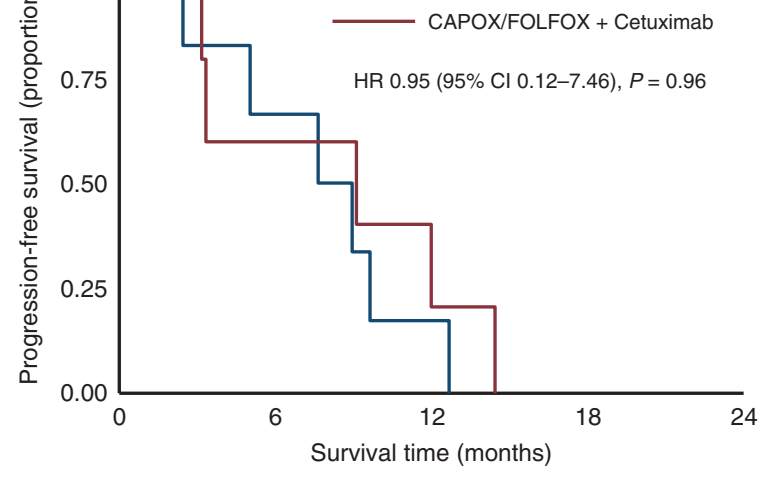

Number at risk

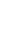

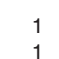

0
0

d

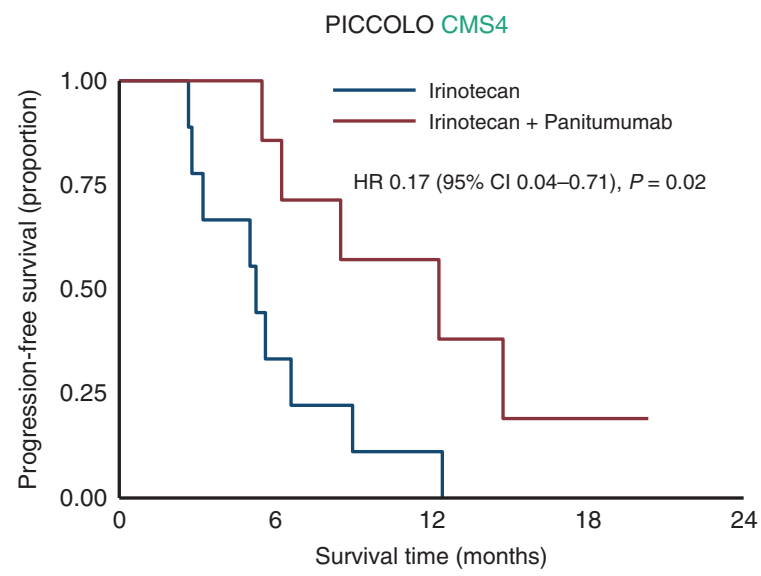

Number at risk

$\square$
$\square$

3
6

1
3

0
1

0

Fig. 4 Molecular subtype-specific efficacy of anti-EGFR in right-sided tumours. Progression-free survival in the COIN trial for CMS2/3 (a) and CMS4 (b) and the PICCOLO trial for CMS2/3 (c) and CMS4 (d). HRs are adjusted for age, sex and WHO performance status. CAPOX capecitabine and oxaliplatin, FOLFOX 5-fluorouracil and oxaliplatin, HR hazard ratio. P-values adjusted for multiple testing: 0.96 (a), 0.96 (b), 0.96 (c), 0.08 (d).

It is important to mention that the CMS classification of both studies was based on primary tumour tissue, which does not take into account any potential subtype switch during progression of disease, as well as the possible impact of chemotherapy on changing tumour biology as we classified the primary tumours pretreatment $[39,40]$.

Despite conducting this analysis on a smaller subcohort of the original trial due to limitations in tissue availability for classification, patient demographic characteristics and outcomes were consistent with the main trial analyses. The small survival benefit in both classified cohorts might be due to the higher number of resected primary tumours, which may be associated with improved OS [41]. This study analysed small subgroups and was underpowered to detect CMS/treatment interactions. Multiple comparisons were made and borderline significant results should be interpreted with caution. The retrospective nature of this study design and the crossstudy comparison does not allow for definitive conclusions about the differential effect of the backbone therapy and anti-EGFR in the different subtypes, especially due to the different lines of therapy (first- versus second-line) which might contribute to the differences found. Nonetheless, we evaluated these two studies with a specific hypothesis in mind, which was confirmed by the result. This positions the current study as a starting point in refining selection criteria for anti-EGFR therapy based on CMS and primary tumour location. Additional studies are warranted to validate these findings and to reach definitive conclusions about the differential effect of chemotherapy backbones in relation with CMSs for anti-EGFR therapy. In addition, our study shows the potential clinical impact of stratifying patients into CMSs. This supports our current effort to implement the CMS classification into everyday clinical practice.

In conclusion, our results suggest that molecular subtypes may play an important role in the effectiveness of the type of chemotherapy backbone given in combination with anti-EGFR therapy. There is some evidence that patients with RAS and BRAF wildtype mesenchymal CMS4 tumours may only benefit when anti-EGFR is combined with irinotecan, even in right-sided primary tumours. For epithelial CMS2/3 tumours, we demonstrate no difference between irinotecan- and oxaliplatin backbones for the beneficial additive effect of anti-EGFR. This may provide the possibility of subtype-specific treatment strategies for the optimal use of anti-EGFR therapy.

\section{DATA AVAILABILITY}

Data used in this study could be provided upon request, only with permission of the authors of the original studies. 


\section{REFERENCES}

1. Van Cutsem E, Kohne CH, Lang I, Folprecht G, Nowacki MP, Cascinu S, et al. Cetuximab plus irinotecan, fluorouracil, and leucovorin as first-line treatment for metastatic colorectal cancer: updated analysis of overall survival according to tumor KRAS and BRAF mutation status. J Clin Oncol. 2011;29:2011-9.

2. Douillard JY, Oliner KS, Siena S, Tabernero J, Burkes R, Barugel M, et al. Panitumumab-FOLFOX4 treatment and RAS mutations in colorectal cancer. $\mathrm{N}$ Engl J Med. 2013;369:1023-34.

3. De Roock W, Claes B, Bernasconi D, De Schutter J, Biesmans B, Fountzilas G, et al. Effects of KRAS, BRAF, NRAS, and PIK3CA mutations on the efficacy of cetuximab plus chemotherapy in chemotherapy-refractory metastatic colorectal cancer: a retrospective consortium analysis. Lancet Oncol. 2010;11:753-62.

4. Vale CL, Tierney JF, Fisher D, Adams RA, Kaplan R, Maughan TS, et al. Does antiEGFR therapy improve outcome in advanced colorectal cancer? A systematic review and meta-analysis. Cancer Treat Rev. 2012;38:618-25.

5. Bokemeyer C, Bondarenko I, Hartmann JT, de Braud F, Schuch G, Zubel A, et al. Efficacy according to biomarker status of cetuximab plus FOLFOX-4 as first-line treatment for metastatic colorectal cancer: the OPUS study. Ann Oncol. 2011; 22:1535-46.

6. Douillard JY, Siena S, Cassidy J, Tabernero J, Burkes R, Barugel M, et al. Randomized, phase III trial of panitumumab with infusional fluorouracil, leucovorin, and oxaliplatin (FOLFOX4) versus FOLFOX4 alone as first-line treatment in patients with previously untreated metastatic colorectal cancer: the PRIME study. J Clin Oncol. 2010;28:4697-705.

7. Tol J, Koopman M, Cats A, Rodenburg CJ, Creemers GJ, Schrama JG, et al. Chemotherapy, bevacizumab, and cetuximab in metastatic colorectal cancer. $\mathrm{N}$ Engl J Med. 2009;360:563-72.

8. Tejpar S, Stintzing S, Ciardiello F, Tabernero J, Van Cutsem E, Beier F, et al Prognostic and predictive relevance of primary tumor location in patients With RAS wild-type metastatic colorectal cancer: retrospective analyses of the CRYSTAL and FIRE-3 trials. JAMA Oncol. 2017;3:194-201.

9. Wang ZX, Wu HX, He MM, Wang YN, Luo HY, Ding PR, et al. Chemotherapy with or without anti-EGFR agents in left- and right-sided metastatic colorectal cancer: an updated meta-analysis. J Natl Compr Canc Netw. 2019;17:805-11.

10. Sunakawa $Y$, Tsuji A, Fujii M, Ichikawa W. No benefit from the addition of antiEGFR antibody in all right-sided metastatic colorectal cancer? Ann Oncol. 2017; 28:2030-1.

11. Guinney J, Dienstmann R, Wang X, de Reynies A, Schlicker A, Soneson C, et al. The consensus molecular subtypes of colorectal cancer. Nat Med. 2015;21:1350-6.

12. De Sousa EMF, Wang X, Jansen M, Fessler E, Trinh A, de Rooij LP, et al. Poorprognosis colon cancer is defined by a molecularly distinct subtype and develops from serrated precursor lesions. Nat Med. 2013;19:614-8.

13. Lenz H-J, Ou F-S, Venook AP, Hochster HS, Niedzwiecki D, Goldberg RM, et al. Impact of consensus molecular subtype on survival in patients with metastatic colorectal cancer: results from CALGB/SWOG 80405 (alliance). J Clin Oncol. 2019;37:1876-85.

14. Trinh A, Trumpi K, De Sousa EMF, Wang X, de Jong JH, Fessler E, et al. Practical and robust identification of molecular subtypes in colorectal cancer by immunohistochemistry. Clin Cancer Res. 2017;23:387-98.

15. Stintzing $S$, Wirapati $P$, Lenz $H J$, Neureiter $D$, Fischer von Weikersthal L, Decker $T$, et al. Consensus molecular subgroups (CMS) of colorectal cancer (CRC) and firstline efficacy of FOLFIRI plus cetuximab or bevacizumab in the FIRE3 (AIO KRK0306) trial. Ann Oncol. 2019;30:1796-803.

16. Aderka D, Stintzing $S$, Heinemann V. Explaining the unexplainable: discrepancies in results from the CALGB/SWOG 80405 and FIRE-3 studies. Lancet Oncol. 2019;20:e274-e83.

17. Maughan TS, Adams RA, Smith CG, Meade AM, Seymour MT, Wilson RH, et al Addition of cetuximab to oxaliplatin-based first-line combination chemotherapy for treatment of advanced colorectal cancer: results of the randomised phase 3 MRC COIN trial. Lancet 2011;377:2103-14.

18. Seymour MT, Brown SR, Middleton G, Maughan T, Richman S, Gwyther S, et al. Panitumumab and irinotecan versus irinotecan alone for patients with KRAS wildtype, fluorouracil-resistant advanced colorectal cancer (PICCOLO): a prospectively stratified randomised trial. Lancet Oncol. 2013;14:749-59.

19. Ten Hoorn S, Trinh A, de Jong J, Koens L, Vermeulen L. Classification of colorectal cancer in molecular subtypes by immunohistochemistry. Methods Mol Biol. 2018; 1765:179-91.

20. Pollard KS, Gilbert HN, Ge Y, Taylor SE, Dudoit S. Multtest: resampling-based multiple hypothesis testing. $\mathrm{R}$ package version $2.46 .0 ; 2021$. Available at: multtest. pdf (bioconductor.org). 2021.

21. Coebergh Van Den Braak RRJ, Ten Hoorn S, Sieuwerts AM, Tuynman JB, Smid M, Wilting SM, et al. Interconnectivity between molecular subtypes and tumor stage in colorectal cancer. BMC Cancer. 2020;20:850.
22. Chan DL, Pavlakis N, Shapiro J, Price TJ, Karapetis CS, Tebbutt NC, et al. Does the chemotherapy backbone impact on the efficacy of targeted agents in metastatic colorectal cancer? A systematic review and meta-analysis of the literature. PLoS ONE 2015:10:e0135599.

23. Ocvirk J, Brodowicz T, Wrba F, Ciuleanu TE, Kurteva G, Beslija S, et al. Cetuximab plus FOLFOX6 or FOLFIRI in metastatic colorectal cancer: CECOG trial. World J. Gastroenterol. 2010;16:3133-43.

24. Okita A, Takahashi S, Ouchi K, Inoue M, Watanabe M, Endo $M$, et al. Consensus molecular subtypes classification of colorectal cancer as a predictive factor for chemotherapeutic efficacy against metastatic colorectal cancer. Oncotarget 2018; 9:18698-711.

25. Brule SY, Jonker DJ, Karapetis CS, O'Callaghan CJ, Moore MJ, Wong R, et al. Location of colon cancer (right-sided versus left-sided) as a prognostic factor and a predictor of benefit from cetuximab in NCIC CO.17. Eur. J. Cancer 2015;51:1405-14.

26. Heinemann V, Modest DP, Weikersthal LFv, Decker T, Kiani A, Vehling-Kaiser U, et al. Gender and tumor location as predictors for efficacy: influence on endpoints in first-line treatment with FOLFIRI in combination with cetuximab or bevacizumab in the AIO KRK 0306 (FIRE3) trial. J Clin Oncol. 2014;32:3600.

27. Ishida T, Ishii $Y$, Tsuruta M, Okabayashi $K$, Akimoto $S$, Koishikawa $K$, et al. Cetuximab promotes SN38 sensitivity via suppression of heat shock protein 27 in colorectal cancer cells with wild-type RAS. Oncol. Rep. 2017;38:926-32.

28. Liu X, Guo WJ, Zhang XW, Cai X, Tian S, Li J. Cetuximab enhances the activities of irinotecan on gastric cancer cell lines through downregulating the EGFR pathway upregulated by irinotecan. Cancer Chemother Pharmacol. 2011;68:871-8.

29. Prewett MC, Hooper AT, Bassi R, Ellis LM, Waksal HW, Hicklin DJ. Enhanced antitumor activity of anti-epidermal growth factor receptor monoclonal antibody IMC-C225 in combination with irinotecan (CPT-11) against human colorectal tumor xenografts. Clin. Cancer Res. 2002;8:994-1003.

30. Petitprez A, Larsen AK. Irinotecan resistance is accompanied by upregulation of EGFR and Src signaling in human cancer models. Curr Pharm Des. 2013;19: 958-64.

31. Cunningham D, Humblet $Y$, Siena $S$, Khayat $D$, Bleiberg $H$, Santoro $A$, et al Cetuximab monotherapy and cetuximab plus irinotecan in irinotecan-refractory metastatic colorectal cancer. N Engl J Med. 2004;351:337-45.

32. Lv ZC, Ning JY, Chen HB. Efficacy and toxicity of adding cetuximab to chemotherapy in the treatment of metastatic colorectal cancer: a meta-analysis from 12 randomized controlled trials. Tumour Biol. 2014;35:11741-50.

33. van Helden EJ. Menke-van der Houven van Oordt CW, Heymans MW, Ket JCF, van den Oord R, Verheul HMW. Optimal use of anti-EGFR monoclonal antibodies for patients with advanced colorectal cancer: a meta-analysis. Cancer Metastasis Rev. 2017:36:395-406.

34. Borelli B, Fontana E, Giordano M, Antoniotti C, Bergamo F, Murgioni S, et al Consensus molecular subtypes and CRCassigner classifications in metastatic colorectal cancer (mCRC): prognostic and predictive impact in the TRIBE2 study. J Clin Oncol. 2020;38:4016.

35. Del Rio M, Mollevi C, Bibeau F, Vie N, Selves J, Emile JF, et al. Molecular subtypes of metastatic colorectal cancer are associated with patient response to irinotecan-based therapies. Eur J Cancer 2017;76:68-75.

36. Yuki S, Gamoh M, Denda T, Takashima A, Takahashi S, Nakamura M, et al. Analysis of consensus molecular subtypes (CMS) classification in the TRICOLORE trial: A randomized phase III trial of S-1 and irinotecan (IRI) plus bevacizumab (Bmab) versus mFOLFOX6 or CapeOX plus Bmab as first-line treatment for metastatic colorectal cancer (mCRC). J Clin Oncol. 2020;38:169.

37. Sadanandam A, Lyssiotis CA, Homicsko K, Collisson EA, Gibb WJ, Wullschleger S, et al. A colorectal cancer classification system that associates cellular phenotype and responses to therapy. Nat Med. 2013;19:619-25.

38. Sveen A, Bruun J, Eide PW, Eilertsen IA, Ramirez L, Murumagi A, et al. Colorectal cancer consensus molecular subtypes translated to preclinical models uncover potentially targetable cancer cell dependencies. Clin Cancer Res. 2018;24: 794-806.

39. Schlicker A, Ellappalayam A, Beumer IJ, Snel MHJ, Mittempergher L, Diosdado B, et al. Investigating the concordance in molecular subtypes of primary colorectal tumors and their matched synchronous liver metastasis. Int J Cancer 2020;147: 2303-15.

40. Trumpi K, Ubink I, Trinh A, Djafarihamedani M, Jongen JM, Govaert KM, et al. Neoadjuvant chemotherapy affects molecular classification of colorectal tumors. Oncogenesis 2017;6:e357.

41. t Lam-Boer J, Van der Geest LG, Verhoef C, Elferink ME, Koopman M, de Wilt JH. Palliative resection of the primary tumor is associated with improved overall survival in incurable stage IV colorectal cancer: A nationwide population-based propensity-score adjusted study in the Netherlands. Int J Cancer 2016;139: 2082-94. 
1088

\section{ACKNOWLEDGEMENTS}

Not applicable.

\section{AUTHOR CONTRIBUTIONS}

$\mathrm{SH}, \mathrm{CP}$ and LV designed the study. TM, JS, MS, PQ, RA and SR provided the patient samples. SH performed the immunohistochemistry stainings and AT and $\mathrm{SH}$ classified the samples. SH, FE and DF performed data analyses. SH, DS, TB, LK, JS, RA, SR, CP and LV wrote the manuscript.

\section{FUNDING INFORMATION}

This work is supported by the Dutch Cancer Society (KWF, 10529) to LV and CP. The European Research Council (ERG-StG 638193), ZonMw (Vidi 016.156.308), and Innovatiefonds Zorgverzekeraars (B17-140) to LV. L.V is a New York Stem Cell Foundation-Robertson Investigator. The COIN trial was funded by Cancer Research UK with an educational grant from Merck KGaA. The PICCOLO trial was also funded by Cancer Research UK with an grant from Amgen Inc.

\section{ETHICS APPROVAL AND CONSENT TO PARTICIPATE}

Both the COIN and the PICCOLO trial obtained written informed consent from all patients to participate in the studies. Both trials were performed in accordance with the Declaration of Helsinki.

\section{CONSENT TO PUBLISH}

Not applicable.

\section{COMPETING INTERESTS}

LV received speaker and consultancy fees from Genentech, Bayer, Servier, Boehringer-Ingelheim, MSD and Pierre Fabre. LV received unrestricted grants from Novartis, Servier, Roche and Roche Diagnostics. LV declares ongoing collaborations with Firalis, LeadPharma and Genentech. RA received speaker and consultancy fees form Amgen, Bayer, Astra Zeneca, Merck. TM received unrestricted grants and speaker fees from Merck KGaA, and unrestricted grants and consultancy fees from AstraZeneca. CP reports an advisory role for Nordic Pharma. PQ has research funding with Roche, GeneFirst and Amgen, previous research funding from Halio, consultancy with Nordlai-Adlyte and advisory boards with Merck, Amgen and Roche. JS received speaker fees from Merck Serono; advisory board-Pierre Fabre, Roche; CME-Gl Connect. No disclosures were reported by the other authors. None of the funders had any role in study design, data collection and analysis, decision to publish, or preparation of the manuscript.

\section{ADDITIONAL INFORMATION}

Supplementary information The online version contains supplementary material available at https://doi.org/10.1038/s41416-021-01477-9.

Correspondence and requests for materials should be addressed to L.V.

Reprints and permission information is available at http://www.nature.com/ reprints

Publisher's note Springer Nature remains neutral with regard to jurisdictional claims in published maps and institutional affiliations.

\begin{abstract}
cc) (i) Open Access This article is licensed under a Creative Commons Attribution 4.0 International License, which permits use, sharing, adaptation, distribution and reproduction in any medium or format, as long as you give appropriate credit to the original author(s) and the source, provide a link to the Creative Commons license, and indicate if changes were made. The images or other third party material in this article are included in the article's Creative Commons license, unless indicated otherwise in a credit line to the material. If material is not included in the article's Creative Commons license and your intended use is not permitted by statutory regulation or exceeds the permitted use, you will need to obtain permission directly from the copyright holder. To view a copy of this license, visit http://creativecommons. org/licenses/by/4.0/.
\end{abstract}

(c) The Author(s) 2021 